\title{
International publications of authors from Bosnia and Herzegovina in Current Contents indexed publications in the first half of $2012^{\star}$
}

\author{
Avdibegović E, Hasanović M, Hodžić M, Selimbašić \\ Z. Psychological symptoms among workers \\ employed in companies undergoing privatization \\ in postwar Bosnia and Herzegovina. Coll Antropol. \\ 2011 Dec;35(4):993-9.
}

Tuzla University Clinical Center, Clinic of Psychiatry, Tuzla, Bosnia and Herzegovina.

In Central and Eastern European countries, after abandoning communism, significant political, economic and social changes occurred, followed by the increase in income inequality and social disparity. The goal of this study was to examine the relationship between psychological symptoms and monthly income of employees in companies undergoing privatization. The study included 258 workers from seven companies undergoing privatization in the Tuzla Canton region. For the study purposes, the Brief Symptom Inventory (BSI) and a general questionnaire with questions about socio-demographic characteristics, income, and workplace, were used. Monthly income of the majority of workers ( 207 or $80.2 \%$ ) was below the monthly income in Bosnia and Herzegovina. Workers with salaries below the average salary for Bosnia and Herzegovina have pronounced somatization, anxiety, paranoia, interpersonal sensitivity and hostility. The BSI scale yielded significant negative correlation between the level of monthly salary and the expression of psychological symptoms $(r=-0.184, p=0.002)$ and between the level of family income and the expression of psychological symptoms $(r=-0.123, p=0.024)$. Based on the study results, it was determined that socio-economic factors such as the level of salary and total family income and job insecurity, educational level, marital status and gender may be predictors of psychological symptoms.

\section{Beganović A, Sefić-Pašić I, Skopljak-Beganović A, Kristić S, Šunjić S, Mekić A, Gazdić-Šantić M, Drljević A, Samek D. Doses to Skin During Dynamic Perfusion Computed Tomography of the Liver. Radiat Prot Dosimetry. 2012 Jun 22. [Epub ahead of print] \\ Department of Medical Physics and Radiation Safety, Clinical Centre of Sarajevo University, Bolnička 25, Sarajevo, Bosnia and Herzegovina.}

Many new computed tomography (CT) techniques have been introduced during the recent years, one of them being CT-assisted dynamic perfusion imaging (perfusion CT, PCT). Many concerns were raised when first cases of deterministic radiation effects were reported. This paper shows how radiochromic films can be utilised as passive dosemeters for use in PCT. Radiochromic dosemeters undergo a colour change directly and do not require chemical processing. Prior to their use, they need to be calibrated. Films are placed on top and on the right side of the patient and exposed during the procedure. Readout is performed using a densitometer. Results show that average local skin doses are $0.51 \pm 0.07$ and $0.42 \pm 0.04 \mathrm{~Gy}$ on top and on the lateral side of the patient, respectively. Results of the patient dosimetry (local skin doses) are consistent. This is due to the fact that each patient had the

${ }^{\star}$ Data for this survey were collected from PubMed database using the keywords Bosnia and Herzegovina and 2012. 
same CT protocol used for imaging $(120 \mathrm{kV}, 60 \mathrm{~mA}$ and $\mathrm{C}(\mathrm{vol})$ of $247.75 \mathrm{mGy})$. Radiochromic films designed for interventional radiology can be effectively used for local skin dose measurements in perfusion CT. Dose values obtained are below the threshold needed for deterministic effects (erythema, hair loss, etc.). These effects might happen if inappropriate CT protocol is used; one that is usually used for routine imaging.

Bralić I, Tahirović H, Matanić D, Vrdoljak O, Stojanović-Špehar S, Kovačić V, BlažekovićMilaković S. Association of early menarche age and overweight/obesity. J Pediatr Endocrinol Metab. 2012;25(1-2):57-62.

Specialist Office in Pediatrics, School of Medicine, University of Split, Trogir, Croatia; Academy of Sciences and Arts of Bosnia and Herzegovina, Sarajevo, Bosnia and Herzegovina; Department for Research and Education, University Clinical Center Tuzla, Tuzla, Bosnia and Herzegovina; City of Zagreb, Zagreb, Croatia; University Hospital "Sestre Milosrdnice”, Children's Hospital Zagreb, Zagreb, Croatia; Department of Family Medicine, "Andrija Stampar" School of Public Health Medical School, University of Zagreb, Zagreb, Croatia; School of Medicine, University of Split, Split, Croatia

AIM: The aim of the study is to assess the association of overweight/obesity and early menarcheal age. PATIENTS AND METHODS: The study comprised 2127 healthy girls aged 9 to 16 years. Menarcheal age was estimated by status quo method. The girls' body weight and height were measured and their body mass index (BMI) calculated. The diagnostic criteria of the WHO were used to define overweight and obesity. Girls with a BMI in the range of 1-2 for age and sex were considered overweight. Girls with a BMI $>2$ standard deviation (SD) for age and sex were considered obese. Girls with a BMI $>1$ SD for age and sex were considered overweight/obese. Social and economic status was analyzed according to years of education completed, parents' occupations, and the number of children in the family. RESULTS: Median menarcheal age was 12.83 years; $25 \%$ girls had menarche before 11.98 years and $75 \%$ by 13.69 years. By 11.21 years, $10 \%$ of girls had had menarche, and $95 \%$ by 14.91 years. Girls who had menarche before 11.98 years had higher body weight values ( 48.5 vs. $40.2 \mathrm{~kg})(\mathrm{p}<0.001)$, height (159.3 vs. $149.2 \mathrm{~cm})(\mathrm{p}<0.001)$, and BMI $(18.9$ vs. $17.8 \mathrm{~kg} / \mathrm{m} 2)(\mathrm{p}=0.003)$ than their peers without menarche. Girls with menarche before 11.98 years had significantly higher BMI values than girls with menarche after 13.69 years (18.94 vs. $17.84 \mathrm{~kg} / \mathrm{m} 2$ ) $(\mathrm{p}=0.008)$. Girls with menarche before 11.98 years and those after 13.69 years differ significantly in distribution of thinness (3.4\% vs. $2.54 \%$ ), normal weight
(85.3\% vs. $91.8 \%)$, and overweight/obesity (11.2\% vs. $5.7 \%)(p=0.002)$. CONCLUSIONS: Girls who experienced early menarche are significantly more often overweight/obese. Overweight/obesity may be considered as one of the predictors for the early occurrence of menarche.

\section{Ćavar M, Sekulić D, Čuljak Z. Complex Interaction of Religiousness with other Factors in Relation to Substance Use and Misuse Among Female Athletes. J Relig Health. 2012 Jun;51(2):381-9.}

Faculty of Science, Mathematics and Education, University of Mostar, Mostar, Bosnia and Herzegovina.

Strength of religious faith (SRF) is rarely studied as a protective factor against substance use and misuse in sports. Herein, we studied the potential buffering effect of the complex socio-educational, sports, and religiousness factors in the protection against substance use and misuse, including cigarettes, analgesics, appetite suppressants, potential doping behavior, and binge drinking. The sample of subjects included 40 high-class female athletes (22-26 years of age). Using a strictly anonymous questionnaire, we investigated different social, educational, and sports factors (including SRF measured by the Santa Clara Strength of Religious Faith Questionnaire) in relation to substance use and misuse. Following the calculation of simple correlations, multiple regression analysis revealed that in combination with low sports experience, SRF has a significant buffering effect against binge alcohol drinking and consumption of appetite suppressants. The data are discussed in comparison with previous findings and theoretical background. Future studies should study the topic while observing samples of recreational and competitive athletes of both genders.

\section{De Cock RF, Knibbe CA, Kulo A, de Hoon \\ J, Verbesselt R, Danhof M, Allegaert K. Developmental pharmacokinetics of propylene glycol in preterm and term neonates. $\mathrm{Br} \mathrm{J}$ Clin Pharmacol. 2012 Apr 27. doi: 10.1111/j.1365- 2125.2012.04312.x. [Epub ahead of print]}

Division of Pharmacology, LACDR, Leiden University, Leiden, The Netherlands; Department of Clinical Pharmacy, St. Antonius Hospital, Nieuwegein, The Netherlands; Centre for Clinical Pharmacology, University Hospitals Leuven, Leuven, Belgium; Institute of Pharmacology, Clinical Pharmacology and Toxicology, Faculty of Medicine, University of Sarajevo, Sarajevo, Bosnia Herzegovina; Neonatal Intensive Care Unit, University Hospitals Leuven, Leuven, Belgium.

Aim: Propylene glycol (PG) is often applied as an excipient in drug formulations. As these formulations 
may also be used in neonates, the aim of this study was to characterize the pharmacokinetics of propylene glycol, co-administered intravenously with paracetamol (800mgPG/1000mg paracetamol) or phenobarbital (700mgPG/200mg phenobarbital) in preterm and term neonates. Methods: A population pharmacokinetic analysis was performed based on 372 PG plasma concentrations from 62 (pre)term neonates (birth weight (Bbw) 630-3980g, postnatal age (PNA) 1-30days) using NONMEM 6.2. The model was subsequently used to simulate PG exposure upon administration of paracetamol or henobarbitalin neonates (gestational age 24-40 weeks). Results: In a one compartment model, birth weight and PNA were both identified as covariates for PG clearance using an allometric function (CL(i) $=0.0849 x\{(\mathrm{BWb} / 2720)(1.69) \mathrm{x}(\mathrm{PNA} / 3)(0.201)\})$. Volume of distribution scaled allometrically with current bodyweight $(\mathrm{V}(\mathrm{i})=0.967 \mathrm{x}\{(\mathrm{BW} / 2720)(1.45)\})$, and was estimated 1.77 times higher when co-administered with henobarbital compared to paracetamol. By introducing these covariates a large part of the interindividual variability on clearance (65\%) as well as on volume of distribution (53\%) was explained. The final model shows that for commonly used dosing regimens, the population mean PG peak and trough concentrations ranges between 33-144 and 28-218 mg/L (peak) and 19-109 and 6-112 mg/L (trough) depending on birth weight and age of the neonates for paracetamol and henobarbital formulations, respectively. Conclusion: A pharmacokinetic model was developed for PG co-administered with paracetamol or phenobarbital in neonates. As such, large variability in PG exposure may be expected in neonates which are dependent on birth weight and postnatal age.

Jelavić B, Grgić M, Čupić H, Kordić M, Vasilj M, Baudoin T. Prognostic value of Helicobacter pylori sinonasal colonization for efficacy of endoscopic sinus surgery. Eur Arch Otorhinolaryngol. 2012 Jan 12. [Epub ahead of print]

Department of Otorhinolaryngology, Mostar University Hospital, Bijeli brijeg b.b, Mostar, Bosnia and Herzegovina.

Compared with rhinologic patients without chronic rhinosinusitis (CRS), a higher prevalence of sinonasal Helicobacter pylori (HP) in patients with CRS was found. This study investigated if HP sinonasal colonization has a prognostic value for efficacy of functional endoscopic sinus surgery (FESS). Nasal polyps of 40 patients with CRS, undergoing FESS, were analyzed for presence of HP using immunohistochemistry (IHC). Patients were categorized as to whether the IHC was positive ( $\mathrm{HP}+$ group) or negative (HPgroup). $\mathrm{HP}+$ group and $\mathrm{HP}$ - group were compared according to the nasal polyp eosinophil density, and to the improvement (difference between pre- and postoperative scores) of the subjective symptom scores, and the nasal endoscopic scores. Nasal polyps in 28 (70\%) patients were positive for HP. There were no significant differences between $\mathrm{HP}+$ group and $\mathrm{HP}$ group comparing the eosinophils, and the improvement of the single symptom and the total symptom scores. HP+ group had significantly greater improvement of the nasal endoscopic scores $(\mathrm{F}[1.38]=6.212$; $\mathrm{P}=0.017)$. There is no influence of sinonasal HP on tissue eosinophilia and on CRS symptoms. There is a prognostic value for endonasal findings: CRS patients with HP have statistically significant greater improvement of the postoperative endoscopic scores.

\section{Jovanović P, Salkić N, Zerem E, Ljuca F. Gammaglutamyl transaminase in biliary obstruction. Eur J Intern Med. 2012 Apr;23(3):e76. Epub 2012 Jan 5.}

No abstract available.

\section{Jurić S, Mišmaš A, Mihić N, Barać AM, Habek $M$. Newly onset sinus bradycardia in the context of multiple sclerosis relapse. Intern Med. 2012;51(9):1121-4. Epub 2012 Apr 29.}

Department of Neurology, University Hospital Mostar, Bosnia and Herzegovina.

Cardiovascular disorders in acute multiple sclerosis (MS) relapse have been infrequently reported. We present a young multiple sclerosis patient with acute onset of cerebellar symptomatology along with sinus bradycardia. Brain magnetic resonance imaging showed one lesion in the left cerebellar hemisphere which showed postcontrast enhancement and one in the midbrain without postcontrast enhancement. No cardiac pathology was found and symptoms gradually improved after a 5-day course of corticosteroid therapy. It is important to bear in mind the possibility of these rare cardiac symptoms in MS patients, because of their timely recognition and appropriate treatment.

Kulo A, de Hoon JN, Allegaert K. The propylene glycol research project to illustrate the feasibility and difficulties to study toxicokinetics in neonates. Int J Pharm. 2012 May 26. [Epub ahead of print]

Center for Clinical Pharmacology, University Hospitals Leuven, Leuven, Belgium; Institute of Pharmacology, Clinical Pharmacology and Toxicology, Faculty of Medicine, University of Sarajevo, Sarajevo, Bosnia and Herzegovina.

This paper aims to describe our propylene glycol (PG) research project to illustrate the feasibility and the dif- 
ficulties encountered to perform excipient studies in neonates. PG is frequently co-administered excipient. PG accumulation potentially results in hyperosmolarity, lactic acidosis or hepato-renal toxicity in adults, reflecting issues related to pharmacokinetics (PKs) and -dynamics (PDs). Consequently, similar observations in neonates are urgently needed. Since newborns display 'physiological' impaired hepatic and renal elimination capacity, description of PG PK in neonates is warranted. The PG PD was assessed based on indicators of renal, hepatic and metabolic (in)tolerance earlier reported in adults and relating to osmolar changes. Based on the PK and PD data collected in neonates, we suggest that there is a lower limit of PG tolerance in neonates. In addition to preliminary data on PG disposition and tolerance in neonates, we mainly focus on the limitations of the current observations and the difficulties encountered during this PG project to further illustrate the specific setting of neonatal research.

Lala C, Framme C, Wolf-Schnurrbusch UE, Wolf S. Three-year results of visual outcome with disease activity-guided ranibizumab algorithm for the treatment of exudative age-related macular degeneration. Acta Ophthalmol. 2012 Jun 14. doi: 10.1111/j.1755-3768.2012.02457.x. [Epub ahead of print]

Universitätsklinik für Augenheilkunde, University of Bern, Bern, Switzerland; Clinical Center of Eastern Sarajevo, Eye Clinic 'Kasindo', E. Sarajevo, Bosnia and Herzegovina.

Purpose: To evaluate 3-year follow-up treatment outcomes with ranibizumab (Lucentis $\left({ }^{\oplus}\right)$ ) $0.5 \mathrm{mg}$ administered either monthly or quarterly on a pro re nata (PRN) basis according to a disease activityguided monitoring and treatment algorithm. Methods: A total of 316 treatment-naive eyes of 316 patients with exudative age-related macular degeneration met the criteria for inclusion in this retrospective, interventional case series. Patients were treated with ranibizumab $0.5 \mathrm{mg}$ according to a disease activityguided algorithm with monthly monitoring. Optical coherence tomography and fluorescein angiography were routinely used to assess disease activity: active lesions were treated with a series of three monthly injections, whereas inactive lesions were treated with quarterly injections. Results: Mean Early Treatment Diabetic Retinopathy Study best-corrected visual acuity improved from 52 letters at baseline to 59 letters at 12 months, achieved with a mean of 7.1 injections, 61 letters at 24 months with a mean of 5.0 injections administered in the second year and 60 letters at 36 months with a mean number of 5.2 injections. Conclusions: Monthly visits and a morphology-driv- en PRN regimen with 3 injections in case of recurrence plus quarterly injections in case of inactive $\mathrm{CNV}$ resulted in an average VA gain of 7-9 letters that could be maintained over 3 years.

\section{Marković-Peković V, Grubiša N. Self-medication with antibiotics in the Republic of Srpska community pharmacies: pharmacy staff behavior. Pharmacoepidemiol Drug Saf. 2012 Feb 15. doi: 10.1002/pds.3218. [Epub ahead of print]}

Ministry of Health and Social Welfare, the Republic of Srpska, Bosnia and Herzegovina.

PURPOSE: Self-medication with antibiotics adds to the global risk of increased spread of bacterial resistance. Attitudes and behavior of health professionals also may reinforce self-medication with antibiotics. The aim of this study was to determine whether selfmedication with antibiotics is possible in our community pharmacies and to what extent, and to evaluate the behavior and service of pharmacy health professionals regarding non-prescription antibiotic dispensation. METHODS: An observational, cross-section study was conducted, and pseudo-patient methodology was used to establish the kind of professional service provided in case of patient's explicit demand to buy an antibiotic for treatment of self-diagnosed upper respiratory tract infection. RESULTS: Of the total 318 community pharmacies, 131 (41\%) were visited and included in the study. Non-prescription antibiotics were dispensed in 76 (58\%) pharmacies. Counseling and symptomatic therapy was offered in 88 (67\%) pharmacies. In $25 \%$ of pharmacies, no symptomatic therapy was offered; instead, only an antibiotic was sold. Amoxicillin was sold in $85 \%$ of cases and, mostly, the one of 1.30 Euro per pack. Both oral and written use instructions were given in $78 \%$ cases, whereas none was given in $3 \%$ of cases. CONCLUSIONS: Selfmedication with antibiotics occurs in our community pharmacies, despite being illegal. Pharmacy staff behavior can be a factor that puts patients at risk for selfmedication with antibiotics. Community pharmacies are failing their tasks in enhancing rational use of antibiotics. Such a practice may be a consequence of weak enforcement and control over the legislation and professional standards.

Mitrović J. Jožef Stefan and the dissolutiondiffusion phenomena--not only a historical note. Int J Pharm. 2012 Jul 15;431(1-2):12-5. Epub 2012 Apr 13.

Faculty for Production and Management Trebinje, University in East Sarajevo, Republic of Srpska, Bosnia and Herzegovina. 
In a series of papers published from 1871 to 1889 , Jožef (Josef) Stefan dealt with several diffusion processes, including also multicomponent systems. In his last paper on diffusion, which appeared in 1889 , he studied the dissolution-diffusion process with a moving interface, and gave an analytical solution to this problem. However, Stefan's dissolution-diffusion analysis is not mentioned in literature, and its existence seems to be unknown in scientific community. The present paper summarizes the main Stefan ideas on dissolution of solids governed by diffusion of solute in the adjacent solvent phase thus making his results accessible to wider scientific circles.

Mladenović I, Jović N, Čutović T, Mladenović G, Kozomara R. Temporomandibular disorders after orthognathic surgery in patients with mandibular prognathism with depression as a risk factor. Acta Odontol Scand. 2012 Feb 9. [Epub ahead of print]

Department of Prosthodontics, Faculty of Medicine, University of East Sarajevo, Bosnia Herzegovina.

Objective. To examine the prevalence of temporomandibular disorders (TMD) after orthodontic-surgical treatment in patients with mandibular prognathism and analyze psychosocial variables related to TMD. Materials and methods. The case-control study comprised 40 patients with mandibular prognathism who underwent combined orthodontic-surgical treatment (orthognathic surgery group). Forty-two patients with untreated mandibular prognathism served as a control group. Research diagnostic criteria for temporomandibular disorders was used in order to assess the clinical diagnosis of TMD (Axis I) and to estimate depression, somatization and patient's disability related to chronic pain (Axis II). Results. The overall prevalence of TMD was not significantly different between the groups. Myofascial pain was significantly higher, while arthralgia, arthritis and arthrosis was significantly lower in the orthognathic group compared with the controls ( $90.5 \%$ vs $50.0 \%, 0.0 \%$ vs $27.8 \%$, respectively) ( $\mathrm{p}<0.05)$. Females in orthognathic surgery group showed higher prevalence of TMD $(\mathrm{p}<0.05)$ and myofascial pain $(\mathrm{p}<0.01)$ and increased level of chronic pain $(\mathrm{p}<0.05)$ in comparison with post-operative males. No significant difference in chronic pain, somatization and depression scores was found between investigated groups. With respect to presence of TMD within the groups depression was higher in untreated subjects with dysfunction $(\mathrm{p}<0.05)$. Conclusion. Prevalence of TMD immediately after completion of orthodontic-surgical treatment for mandibular prognathism is similar to frequency of dysfunction in untreated subjects, is significantly higher in females and is most commonly myogenic. Furthermore, females show an increased level of chronic pain post- operatively. Somatization and depression levels do not differ between patients with corrected prognathism and untreated prognathic patients.

Pehar M, Vukoja I, Rozić D, Mišković J.

Spontaneous diaphragmatic rupture related to local invasion by retroperitoneal liposarcoma. Ann R Coll Surg Engl. 2012 Jan;94(1):e18-9.

University Hospital Mostar, Mostar, Bosnia and Herzegovina.

We report a case of the female patient who was admitted to the hospital because of syncope experienced while climbing stairs. Diagnostic workup raised the suspicion of a right diaphragmatic rupture that was eventually confirmed by surgery (right-sided thoracotomy). Surgery also revealed tissue protruding through the rupture site from within the retroperitoneum that was proven subsequently to be a dedifferentiated liposarcoma. Second surgery was performed to completely remove the liposarcoma tissue and repair a coincident old right lumbar region hernia. The patient recovered fully. Spontaneous rupture of the diaphragm is rare and this is especially true for the right hemidiaphragm. We report the first case of diaphragmatic rupture caused by local infiltration by a retroperitoneal liposarcoma. This and similar reports emphasise that in cases with high clinical suspicion of diaphragmatic rupture, diagnosis should be pursued even in the absence of a preceding traumatic event.

\section{Petrić I, Helić A, Avdić EA. Evolution of process parameters and determination of kinetics for co- composting of organic fraction of municipal solid waste with poultry manure. Bioresour Technol. 2012 Aug;117:107-16. Epub 2012 Apr 26.}

Department of Process Engineering, Faculty of Technology, University of Tuzla, Univerzitetska 8, Tuzla, Bosnia and Herzegovina.

This study aimed to monitor the process parameters and to determine kinetics in composting of organic fraction of municipal solid waste (OFMSW) and poultry manure. The experiments were carried out with three different mixtures. The results showed that the mixture $60 \%$ OFMSW, $20 \%$ poultry manure, $10 \%$ mature compost and $10 \%$ sawdust provided the most appropriate conditions for composting process. Using nine kinetic models and nonlinear regression method, kinetic parameters were estimated and the models were analyzed with four statistical indicators. Kinetic models with four measured variables proved to be better than models with less number of measured variables. The number of measured experimental variables influences kinetics more than the number of kinetic 
parameters. Satisfactory fittings of proposed kinetic model to the experimental data of OM were achieved. The model is more suitable for data obtained from composting of mixtures with much higher percentage of OFMSW than percentage of poultry manure.

Ristić S, Lukić L, Maksimović Ž, Marić S, Marić V, Kovačević M, Trifunović D, Pavlović D, Mijatović S, Marinković J, Đukanović L. High prevalence of risk factors for chronic kidney disease in Balkan endemic nephropathy foci. Ren Fail. 2012;34(4):467-71. Epub 2012 Feb 24.

Foča Medical Faculty, University of East Sarajevo, Sarajevo, Bosnia and Herzegovina.

BACKGROUND/AIMS: The aim of this study was to find out the prevalence of the most frequent risk factors for chronic kidney disease (CKD) and the prevalence of urinary abnormalities in adult inhabitants of three Balkan endemic nephropathy (BEN) villages near Bijeljina, Bosnia and Herzegovina. METHODS: The survey consisted of an interview, blood pressure measurement, and urine dipstick test for proteinuria, hematuria, and glycosuria. RESULTS: The study involved 1625 (739 males, aged $51 \pm 16$ years) subjects: 319 (19.6\%) with positive family history for BEN, 585 (36\%) with hypertension, 604 (37.2\%) above 60 years, 146 (9\%) with diabetes, and $566(34.8 \%)$ with none of these risk factors. Proteinuria was present in $6.2-7.1 \%$ of the subjects with risk factors for CKD but in $3.4 \%$ of those without risk factors. Systolic blood pressure and BEN in brother/sister were found to be significant variables associated with proteinuria, but female gender and history of kidney disease with hematuria. CONCLUSION: In addition to a family burden for $\mathrm{BEN}$, other risk factors for CKD were highly prevalent in BEN villages of the Bijeljina municipality. The frequency of proteinuria was higher in the at-risk group than in the group without risk factors and increased with the number of risk factors.

Sekulić D, Ostojić M, Ostojić Z, Hajdarević B, Ostojić Lj. Substance abuse prevalence and its relation to scholastic achievement and sport factors: An analysis among adolescents of the Herzegovina-Neretva Canton in Bosnia and Herzegovina. BMC Public Health. 2012 Apr 5;12(1):274. [Epub ahead of print]

BACKGROUND: Substance abuse among adolescents is a major public health and social problem. However, studies rarely investigate the relationships between substance abuse, educational achievement and sport factors. Substance abuse is an even more significant problem in societies that have experienced trauma, such as Bosnia and Herzegovina, which have had re- cent wars. The aims of this study were to investigate substance abuse among adolescents in Bosnia and Herzegovina and to study the potential gender-specific relationships between a) sport factors (physical activity/exercise/athletic participation) and substance abuse and b) scholastic achievement and substance abuse. METHODS: Our sample consisted of 1,032 adolescents who were 17 to 18 years old ( 435 boys and 597 girls) and who were in the final grade of high school. These subjects were randomly selected from the territory of Herzegovina-Neretva Canton of Bosnia and Herzegovina. Retrospective testing was performed using an extensive self-administered questionnaire. The questionnaire included questions involving topics such as sociodemographic variables, scholastic variables, sport factors, and substance abuse data (smoking habits, drugs consumption and alcohol consumption using the AUDIT questionnaire). Descriptive statistics, frequencies, analyses of the differences and correlational analyses were performed. RESULTS: Our results found that greater than one-third of the boys and one-fourth of the girls were daily smokers, and almost half of the boys and one-fifth of the girls practiced harmful drinking; other drugs (i.e. heroin, cocaine, amphetamines, etc.) were rarely consumed. Boys dominated in sport factors, whereas girls were more successful in scholastic achievement. Approximately $23 \%$ of the boys and $6 \%$ of the girls reported that they practiced harmful drinking and smoked simultaneously. Educational failure, which was defined as having one or more negative grades at the end of the last two school years, was identified in $20 \%$ of the boys and $9 \%$ of the girls. In both genders, substance abuse was negatively correlated with educational achievement, and half of those students who failed educationally reported daily smoking. Among the girls who experienced education failure, $33 \%$ were smokers, and $22 \%$ practiced harmful drinking. Sport factors were weakly correlated with substance abuse in boys; thus, we could not support the hypothesis that sports are a protective factor against substance abuse among male adolescents. In girls, participation in team sports was related with a higher incidence of smoking, but there was no evidence of sport factors having an influence on the consumption of alcohol. CONCLUSION: In this study, the incidence of smoking and the consumption of alcohol were alarmingly high. These findings demonstrate the need for intervention programs to address these issues. These problems are particularly important, considering that substance abuse has a negative impact on educational achievement among boys and girls, and sport factors have not been found to be protective factors against substance abuse.

Smajlović L, Davoren J, Heyman P, Cochez C, Haas C, Maake C, Hukić M. Development and optimization of a PCR assay for detection of 
Dobrava and Puumala hantaviruses in Bosnia and Herzegovina. J Virol Methods. 2012 Jun;182(12):37-42. Epub 2012 Mar 13.

International Commission on Missing Persons, Sarajevo, Bosnia and Herzegovina.

Hantavirus-specific serology tests are the main diagnostic technique for detection of hantavirus infection in Bosnia and Herzegovina. In order to enhance hantavirus infections monitoring a sensitive PCR based assay was developed to detect Dobrava (DOBV) and Puumala (PUUV) hantaviruses. Nested primer sets were designed within three different regions of the viral RNA ( $\mathrm{S}$ and $\mathrm{M}$ segment of DOBV and $\mathrm{M}$ segment of PUUV) based on highly similar regions from a number of different European hantavirus strains. Assay conditions were optimized using cell cultures infected with DOBV Slovenia, PUUV Sotkamo and PUUV CG 18-20. This sensitive and specific assay has proven to be useful for detection of both Puumala and Dobrava hantaviruses.

Šimić D, Šitum M, Marijanović I, Hadžigrahić N. Most common skin tumours in correlation with solar ultraviolet radiation in the area of West Herzegovina. Coll Antropol. 2011 Dec;35(4):1129-34.

University of Mostar, Mostar University Clinical

Hospital, Department for Dermatology and

Venerology, Mostar, Bosnia and Herzegovina

Incidence rate of skin tumours, both, non-melanoma and melanoma, is increasing nowadays. Various etiological factors are of relevance for the occurrence of the diseases. The solar radiation, as well, long-term exposure to ultraviolet (UV) radiation, have the greatest impact on development of these skin tumours. Nonmelanoma skin tumours, Basal Cell Carcinoma (BCC) and Squamous Cell Carcinoma (SCC), are the most common skin tumours in humans, and usually develop on the chronically photo-exposed areas. As for the Malignant Melanoma (MM), one of the most aggressive skin tumours, the exposure to solar radiation also plays an important role. This study investigates the correlation between the skin tumours and UV radiation in the area of West Herzegovina, on the sample of 1676 patients. It presents the occurrence of skin tumours in the period from 1997 to 2003 . The study investigates the incidence and the risk factors separately for every skin tumour which can be etiologically related to the occurrence of skin tumours and UV radiation: occupation, exposure to UV radiation, skin type, and family history on malignan tumours within the patient's family. The exact incidence rate of non-melanoma and melanoma skin tumours in Bosnia and Herzegovina is still unknown, for the reason that the united National Cancer Register does not exist yet.
Tahirović E, Begić H, Tahirović H, Varni JW. Quality of life in children after cardiac surgery for congenital heart disease. Coll Antropol. 2011 Dec;35(4):1285-90.

University of Sarajevo, Sarajevo University Clinical Center, Heart Center, Sarajevo, Bosnia and Herzegovina.

The aim of this study was to assess the quality of life children after cardiac surgery for congenital heart disease (CHD) and to compare these results with healthy children. To assess the quality of life children after surgery for CHD we performed a cross-sectional study of 114 patients who were patients at the Department of Paediatrics in Tuzla, between the ages of 2 and 18 years, of both sexes, and with one of their parents. We used the "PedsQL 4.0 Generic Core Scales", with both child self-report and parent proxy-reports. By self assessment, the PedsQL total scores for quality of life were statistically significantly different between children after cardiac surgery for ages 13 to 18 years and healthy children, while by parent report PedsQL total scores were statistically significantly different between children after cardiac surgery for ages 5 to 7 years and healthy children. By self assessment, children after cardiac surgery for ages from 5 to 7 and 13 to 18 years reported that they have a statistically significantly lower quality of life in the segment school functioning compared to the healthy children. By parental assessment, children after cardiac surgery for ages 2 to 4,5 to 7 and 8 to 12 years have a statistically significantly lower quality of life in the segments of physical and psychosocial health, emotional, social and school functioning. The results of our study indicate that children after cardiac surgery for CHD by self and parent assessment have a lower quality of life than healthy children.

Tahirović H, Toromanović A, Tahirović E, Begić H, Varni JW. Health-related quality of life and metabolic control in children with type 1 diabetes mellitus in Bosnia and Herzegovina. Coll Antropol. 2012 Mar;36(1):117-21.

University Clinical Centre Tuzla, Department for Research and Education, Tuzla, Bosnia and Herzegovina

The primary objective of the study was to examine the relationship between generic and disease-specific HRQOL scores and metabolic control in children with Type 1 Diabetes Mellitus (T1DM). This cross-sectional study included 65 consecutive children between ages 5 and 18 years with T1DM. According to their values of glycosylated hemoglobin $(\mathrm{HbA}(1 \mathrm{C}))$, the children were assigned to one of two groups. In Group $1(\mathrm{~N}$ $=21)$ were the children with $\mathrm{HbA}(1 \mathrm{C})$ values $<8 \%$ 
(good to moderate metabolic control) and Group $2(\mathrm{~N}$ $=44$ ) were children with $>8 \%$ (poor metabolic control). To evaluate generic and disease-specific HRQOL scores in children with T1DM in relation to metabolic control, we used the PedsQL 4.0 Generic Core Scales and the PedsQL 3.0 Diabetes Module. The patients in Group 1, by pediatric patient self-report and parent proxy-report, had statistically better disease-specific HRQOL scores on the diabetes symptoms, treatment barriers, treatment adherence and worry domains in comparison with Group 2. We also found significant correlations between the total generic HRQOL scores and $\mathrm{HbA}(1 \mathrm{C})$ for both parent proxy-reports' Spearman's coefficient of rank correlation rho $=-0.257$; $p$ $=0.0412$ and pediatric patients' Spearman's coefficient of rank correlation rho $=-0.269 ; \mathrm{p}=0.0313$. The current findings suggest that poor glycemic control in children with T1DM is associated with lower generic and disease-specific HRQOL scores in developing and transitional countries.

Vranić S, Bender R, Palazzo J, Gatalica Z. A review of adenoid cystic carcinoma of the breast with emphasis on its molecular and genetic characteristics. Hum Pathol. 2012 Apr 18. [Epub ahead of print]

Department of Pathology, Clinical Center of the University of Sarajevo, Sarajevo, Bosnia and Herzegovina.

Breast carcinomas that do not express estrogen receptor $\alpha$, progesterone receptor, or human epidermal growth factor receptor 2 are frequently grouped together as "triple negative" and considered an aggressive type of breast malignancy; however, this group is not homogeneous. Adenoid cystic carcinoma of the breast is a rare type of breast cancer with such triplenegative features and, generally, a more favorable clinical course. This comprehensive review describes diagnostic, molecular, and clinical features of adenoid cystic carcinoma and compares them with those of triple-negative breast carcinomas of no special type.

Zerem E. Percutaneous versus endoscopic approach in treatment of acute cholecystitis. Gastrointest Endosc. 2012 Jan;75(1):226; author reply 226-7.

No abstract available.

Zerem E, Imamović G, Ljuca F, Alidžanović J. What is the optimal treatment for appendiceal mass formed after acute perforated appendicitis. World J Gastroenterol. 2012 Apr 21;18(15):1849-50.
We read with great interest the editorial article by Meshikhes AWN published in issue 25 of World J Gastroenterol 2011. The article described the advantages of emergency laparoscopic appendectomy compared with interval appendectomy as a new safe treatment modality for the appendiceal mass. The author concluded that the emergency laparoscopic appendectomy was a safe treatment modality for the appendiceal mass, and might prove to be more cost-effective than conservative treatment, with no need for interval appendectomy. However, we would like to highlight certain issues regarding the possibility of percutaneous catheter drainage to successfully treat the appendiceal mass, with no need for appendectomy, too.

\section{Zerem E, Imamović G, Mavija Z. Is irrigation necessary during endoscopic necrosectomy of pancreatic necroses? Surg Endosc. 2012 Apr 26. [Epub ahead of print] \\ Department of Gastroenterology, University Clinical Center Tuzla, Trnovac bb, Tuzla, Bosnia and Herzegovina}

No abstract available.

Zerem E, Omerović S. Can percutaneous cholecystostomy be a definitive management for both acute calculous and acalculous cholecystitis? J Clin Gastroenterol. 2012 Mar;46(3):251.

No abstract available.

Zerem E, Pavlović-Čalić N, Bevanda M. Is minimally invasive retroperitoneal pancreatic necrosectomy too aggressive in treating infected pancreatic necrosis. Pancreatology. 2011;11(6):6101. Epub 2012 Feb 2.

No abstract available.

Zerem E, Sušić A, Pavlović-Čalić N, Haračić B, Jovanović $P$. What is the optimal treatment for peripancreatic fluid collections? J Gastrointest Surg. 2012 Aug;16(8):1635-6. Epub 2012 Feb 4.

Department of Gastroenterology, University Clinical Center Tuzla, Trnovac bb, Tuzla, Bosnia and Herzegovina.

No abstract available.

by Nerma Tanović 